Article

\title{
The Bessel Expansion of Fourier Integral on Finite Interval
}

\author{
Yongxiong Zhou ${ }^{\dagger}$ and Zhenyu Zhao *,+ \\ Faculty of Mathematics and Computer Science, Guangdong Ocean University, Zhanjiang 524088, China; \\ zhouyongxiong@163.com \\ * Correspondence: wozitianshanglai@163.com \\ t These authors contributed equally to this work.
}

Received: 1 April 2019; Accepted: 24 April 2019; Published: 1 May 2019

\begin{abstract}
In this paper, we further extend the Filon-type method to the Bessel function expansion for calculating Fourier integral. By means of complex analysis, this expansion is effective for all the oscillation frequencies. Namely, the errors of the expansion not only decrease as the order of the derivative increases, but also decrease rapidly as the frequency increases. Some numerical experiments are also presented to verify the effectiveness of the method.
\end{abstract}

Keywords: fourier integrals; bessel function; quadrature; Filon-type method

\section{Introduction}

Highly oscillatory problems widely appear in many fields of mathematics and engineering, such as asymptotic analysis and electromagnetics [1-7]. Especially, the Fourier integral $\int_{-1}^{1} f(x) e^{i \omega x} d x$ is often encountered. While the parameter $|\omega| \gg 1$, the integrand is highly oscillatory, and this kind of integral is often called highly oscillatory integral. This kind of integration often appears in high frequency acoustic scattering, which is why we study this kind of problem. However, when using the traditional method for calculating the highly oscillatory integrals, such as Gaussian quadrature rule, usually very small discrete steps have to be taken before they can receive a satisfactory numerical results, which makes the computational cost quite high, thus the traditional numerical integration method usually cannot be used for highly oscillatory integral calculation [3-7]. In 1928, Filon first proposed the Filon method [8], which laid the foundation for the numerical method in the future. In recent years, many numerical analysts have developed various Filon-type methods [3-5]. An excellent reference on this topic is [3]. For a simple but classical example, the Filon-type method can be defined as

$$
\int_{-1}^{1} f(x) e^{i \omega x} d x \approx \int_{-1}^{1} p_{2 n+1}(x) e^{i \omega x} d x, \quad f \in C^{\infty}[-1,1]
$$

where $p_{2 n+1}(x)$ is $2 n+1$ order Hermite interpolation polynomial of $f(x)$ satisfying

$$
p_{2 n+1}^{(k)}(-1)=f^{(k)}(-1), p_{2 n+1}^{(k)}(1)=f^{(k)}(1), \quad k=0,1, \ldots, n .
$$

However, the expression and error analysis of Filon-type method are not clear enough.

Therefore, we extend the Filon-type method to the Bessel function expansion in this paper. In addition, the upper bounds for reminders of the expansions are given by complex analysis.

The structure of the paper is as follows. Section 2 gives some basic theoretical results. The Bessel expansion of normal Fourier integral can be found in Section 3 and the Bessel expansion of Fourier integral with symmetric branch points are shown in Section 4 . The numerical experiments are provided in Section 5 to show the effectiveness of the new method. 


\section{Some Basic Theoretical Results}

We first give some theoretical results in this section. Firstly, we introduce Equations (1) and (2) (also see [9], Equation (8) and [10]).According to $e^{i \omega x}=\sum_{j=0}^{\infty} \frac{(i \omega x)^{j}}{j !}$ and integrating item by item,

$$
\int_{-1}^{1}\left(1-x^{2}\right)^{\alpha} e^{i \omega x} d x=\sum_{j=0}^{\infty} \frac{(i \omega)^{j}}{j !} \int_{-1}^{1}\left(1-x^{2}\right)^{\alpha} x^{j} d x=\Gamma(\alpha+1) \sum_{j=0}^{\infty} \frac{(i \omega)^{j}}{j !} \frac{1+(-1)^{j}}{2} \frac{\Gamma(j / 2+1 / 2)}{\Gamma(\alpha+j / 2+3 / 2)} .
$$

By $m=j / 2$, we have

$$
\int_{-1}^{1}\left(1-x^{2}\right)^{\alpha} e^{i \omega x} d x=\Gamma(\alpha+1) \sum_{m=0}^{\infty} \frac{(i \omega)^{2 m}}{(2 m) !} \frac{\Gamma(m+1 / 2)}{\Gamma(\alpha+m+3 / 2)} .
$$

Thus, according to the series expression of Bessel function of the first kind [11] $J_{\alpha}(x)=$ $\sum_{m=0}^{\infty} \frac{(-1)^{m}}{m ! \Gamma(m+\alpha+1 / 2)}\left(\frac{x}{2}\right)^{2 m+\alpha}$, and $\frac{\Gamma(m+1 / 2)}{(2 m) !}=\frac{\sqrt{\pi}}{4^{m} m !}$, if $\omega \neq 0$, we have

$$
\int_{-1}^{1}\left(1-x^{2}\right)^{\alpha} e^{i \omega x} d x= \begin{cases}\sqrt{\pi} \Gamma(\alpha+1)(-2 / \omega)^{\alpha+1 / 2} J_{\alpha+1 / 2}(-\omega), & \arg \omega=\pi, \\ \sqrt{\pi} \Gamma(\alpha+1)(2 / \omega)^{\alpha+1 / 2} J_{\alpha+1 / 2}(\omega), & \arg \omega \neq \pi .\end{cases}
$$

We only focus on the case of $\omega>0$ for no segmentation. If $\omega>0$, we have

$$
\int_{-1}^{1}\left(1-x^{2}\right)^{\alpha} e^{i \omega x} d x=\sqrt{\pi} \Gamma(\alpha+1)\left(\frac{2}{\omega}\right)^{\alpha+\frac{1}{2}} J_{\alpha+\frac{1}{2}}(\omega), \quad \Re(\alpha)>-1 .
$$

Similarly,

$$
\int_{-1}^{1} x\left(1-x^{2}\right)^{\alpha} e^{i \omega x} d x=i \sqrt{\pi} \Gamma(\alpha+1)\left(\frac{2}{\omega}\right)^{\alpha+\frac{1}{2}} J_{\alpha+\frac{3}{2}}(\omega), \quad \Re(\alpha)>-1 .
$$

Now, we derive some results about Cauchy-type integral kernel, function approximation and its remainder estimation.

Assuming that $U$ is a region, $f$ is analytic in the region $U$ and continuous in the closed region $\bar{U}$, i.e., $f \in \mathcal{O}(U) \cap \mathcal{C}(\bar{U}), V \subset U, \partial U=\Gamma$, and $P$ is the polynomial. We rewrite Cauchy integral formula $f(z)=\frac{1}{2 \pi i} \int_{\Gamma} \frac{f(\zeta)}{\zeta-z} d \zeta$ as

$$
f(z)=\frac{1}{2 \pi i} \int_{\Gamma} f(\zeta) \frac{P(\zeta)-P(z)}{\zeta-z} \frac{1}{P(\zeta)-P(z)} d \zeta
$$

where we define $\frac{1}{P(\zeta)-P(z)}$ as Cauchy-type integral kernel. If $|P(z)|<|P(\zeta)|$ for $z \in V \subset U, \zeta \in \Gamma$, then the geometric series

$$
\frac{1}{P(\zeta)-P(z)}=\sum_{k=0}^{\infty} \frac{P^{k}(z)}{P^{k+1}(\zeta)}
$$

is absolutely and consistently convergent such that we can exchange the order of integration and summation,

$$
f(z)=\sum_{k=0}^{\infty} c_{k}(z) P^{k}(z)
$$

where coefficients $c_{k}(z)=\frac{1}{2 \pi i} \int_{\Gamma} f(\zeta) \frac{P(\zeta)-P(z)}{\zeta-z} \frac{1}{P^{k+1}(\zeta)} d \zeta$. 
Theorem 1. Suppose $f \in \mathcal{O}(U) \cap C(\bar{U})$ and $z \in V \subset U$, then the error of Equation (3) satisfies

$$
\left|f(z)-\sum_{k=0}^{n-1} c_{k}(z) P^{k}(z)\right| \leq \frac{M L}{2 \pi d} q^{n}|P(z)|^{n},
$$

where $L$ is the length of $\Gamma$, the distance $d=\inf \{|z-\zeta| \mid z \in V, \zeta \in \Gamma\}$, and

$$
M=\max _{\zeta \in \Gamma}|f(\zeta)|, \quad q=\max _{\zeta \in \Gamma} \frac{1}{|P(\zeta)|} .
$$

Proof. By the integral value theorem, the conclusion is obviously true according to the formula

$$
f(z)-\sum_{k=0}^{n-1} c_{k}(z) P^{k}(z)=P^{n}(z) \frac{1}{2 \pi i} \int_{\Gamma} \frac{f(\zeta)}{\zeta-z} \frac{1}{P^{n}(\zeta)} d \zeta .
$$

\section{The Bessel Expansion of Normal Fourier Integral}

In this section, we focus on the normal Fourier integral of the form

$$
I=\int_{-1}^{1} f(x) e^{i \omega x} d x, f \in C^{\infty}[-1,1] .
$$

\subsection{Hermite Series of Analytic Functions}

Let $P(z)=1-z^{2}$, then according to Equation (3),

$$
f(z)=\sum_{k=0}^{\infty}\left(1-z^{2}\right)^{k} \frac{-1}{2 \pi i} \int_{\Gamma} \frac{\zeta+z}{\left(1-\zeta^{2}\right)^{k+1}} f(\zeta) d \zeta,
$$

where $\Gamma$ is a Jordan curve [12] with the points \pm 1 in its interior, then we derive the series of $f$,

$$
f(z)=\sum_{k=0}^{\infty}\left(\alpha_{k}+\beta_{k} z\right)\left(1-z^{2}\right)^{k}
$$

and we define it as Hermite series, in which the coefficients are

$$
\alpha_{k}=\frac{-1}{2 \pi i} \int_{\Gamma} \frac{\zeta f(\zeta)}{\left(1-\zeta^{2}\right)^{k+1}} d \zeta, \quad \beta_{k}=\frac{-1}{2 \pi i} \int_{\Gamma} \frac{f(\zeta)}{\left(1-\zeta^{2}\right)^{k+1}} d \zeta,
$$

and they can be computed by the residue theorem. Let

$$
\sigma_{1}(\zeta)=\frac{\zeta f(\zeta)}{(1+\zeta)^{k+1}}, \quad \sigma_{2}(\zeta)=\frac{\zeta f(\zeta)}{(1-\zeta)^{k+1}}
$$

by Taylor expansion,

$$
\sigma_{1}(\zeta)=\sum_{k=0}^{\infty} \frac{\sigma_{1}^{(k)}(1)}{k !}(\zeta-1)^{k}, \quad \sigma_{2}(\zeta)=\sum_{k=0}^{\infty} \frac{\sigma_{2}^{(k)}(-1)}{k !}(\zeta+1)^{k},
$$

then we obtain the coefficients

$$
\alpha_{k}=(-1)^{k} \frac{\sigma_{1}^{(k)}(1)}{k !}-\frac{\sigma_{2}^{(k)}(-1)}{k !} .
$$


Similarly, let

$$
\tau_{1}(\zeta)=\frac{f(\zeta)}{(1+\zeta)^{k+1}}, \quad \tau_{2}(\zeta)=\frac{f(\zeta)}{(1-\zeta)^{k+1}}
$$

we have

$$
\beta_{k}=(-1)^{k} \frac{\tau_{1}^{(k)}(1)}{k !}-\frac{\tau_{2}^{(k)}(-1)}{k !}
$$

According to Theorem 1, we estimate the truncation errors of Equation (5) as follows.

Corollary 1. Suppose $f(z)$ is analytic in the disk $|z|<R, R \geq \sqrt{2}$, then

$$
\left|f(z)-\sum_{k=0}^{n-1}\left(\alpha_{k}+\beta_{k} z\right)\left(1-z^{2}\right)^{k}\right| \leq \frac{R M_{R}}{(R-1)\left(R^{2}-1\right)^{n}}\left|1-z^{2}\right|^{n}, \quad|z| \leq 1 .
$$

where

$$
M_{R}=\max _{|z|=R}|f(z)|
$$

\subsection{The Bessel Expansion of Fourier Integral With Analytic Function}

Theorem 2. Suppose $f(z)$ is analytic in the disk $|z|<R, R \geq \sqrt{2}$, then for $\omega>0,0 \leq a \leq 1$,

$$
\begin{aligned}
\int_{-1}^{1} f(x) e^{i \omega x} d x= & \sum_{k=0}^{n-1} \alpha_{k} \sqrt{\pi} \Gamma(k+1)\left(\frac{2}{\omega}\right)^{k+\frac{1}{2}} J_{k+\frac{1}{2}}(\omega) \\
& +i \sum_{k=0}^{n-1} \beta_{k} \sqrt{\pi} \Gamma(k+1)\left(\frac{2}{\omega}\right)^{k+\frac{1}{2}} J_{k+\frac{3}{2}}(\omega)+\rho_{n} .
\end{aligned}
$$

where the remainder satisfies

$$
\left|\rho_{n}\right| \leq \frac{R M_{R}\left(1+4 a^{2}\right)^{(n+1) / 2}}{(R-1)\left(R^{2}-1\right)^{n}} \int_{-1}^{1}\left(1-t^{2}\right)^{n} e^{-\omega a\left(1-t^{2}\right)} d t .
$$

Proof. The path $\gamma$ is shown in Figure 1, and can be represented as

$$
\gamma=\left\{z \mid z=t+i a\left(1-t^{2}\right),-1 \leq t \leq 1,0 \leq a \leq 1\right\} .
$$

Let

$$
\varrho_{n}(z)=f(z)-\sum_{k=0}^{n-1}\left(\alpha_{k}+\beta_{k} z\right)\left(1-z^{2}\right)^{k},
$$

according to Cauchy theorem, the error estimation

$$
\rho_{n}=\int_{-1}^{1} \varrho_{n}(x) e^{i \omega x} d x=\int_{\gamma} \varrho_{n}(z) e^{i \omega z} d z .
$$

According to the inequality in Equation (6),

$$
\left|\varrho_{n}(z)\right| \leq \frac{R M_{R}}{(R-1)\left(R^{2}-1\right)^{n}}\left|1-z^{2}\right|^{n}, \quad|z| \leq 1,
$$

and $\left|1-z^{2}\right| \leq\left(1+4 a^{2}\right)^{1 / 2}\left(1-t^{2}\right), z \in \gamma$, then

$$
\left|\varrho_{n}(z)\right| \leq \frac{\left(1+4 a^{2}\right)^{n / 2} R M_{R}}{(R-1)\left(R^{2}-1\right)^{n}}\left(1-t^{2}\right)^{n}, \quad z \in \gamma .
$$


In the integral on the right side of Equation (9), since $\left|e^{i \omega z}\right|=e^{-\omega a\left(1-t^{2}\right)},\left|\frac{d z}{d t}\right| \leq\left(1+4 a^{2}\right)^{1 / 2}$ on the path $\gamma$, it is true that

$$
\left|\int_{\gamma} \varrho_{n}(z) e^{i \omega z} d z\right| \leq \frac{R M_{R}\left(1+4 a^{2}\right)^{(n+1) / 2}}{(R-1)\left(R^{2}-1\right)^{n}} \int_{-1}^{1}\left(1-t^{2}\right)^{n} e^{-\omega a\left(1-t^{2}\right)} d t .
$$

According to Equations (1) and (2), the conclusion is true.

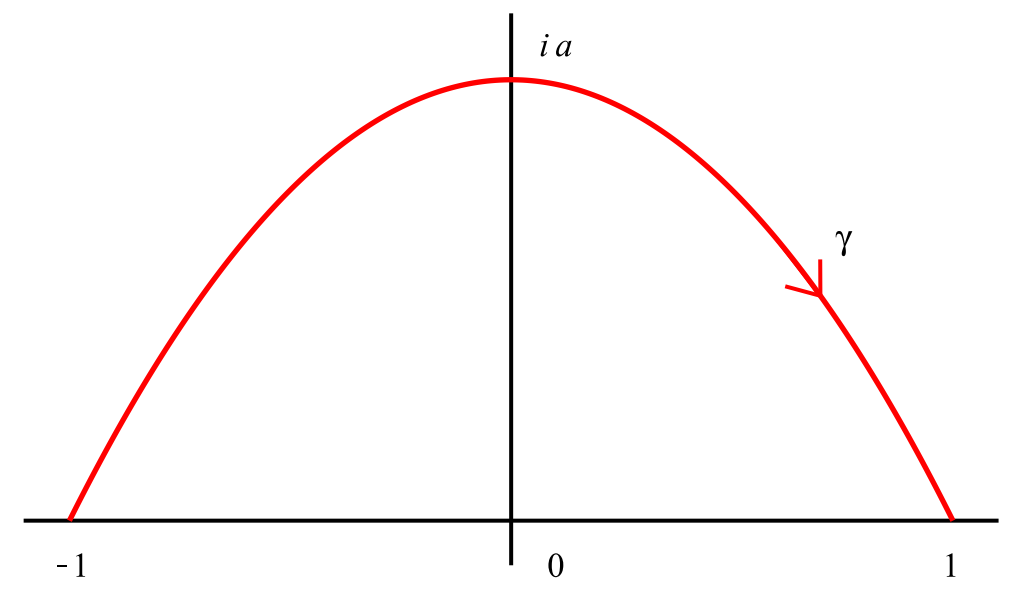

Figure 1. The homotopy deformation of integral path.

Letting $a=0$, the right side of the inequality in Equation (8) behaves as

$$
\text { const } \frac{1}{\sqrt{n}\left(R^{2}-1\right)^{n}} \rightarrow 0, \quad \text { for } n \rightarrow \infty,
$$

then we have the following.

Corollary 2. Under the assuming condition in Theorem 2, it is true that

$$
\begin{aligned}
\int_{-1}^{1} f(x) e^{i \omega x} d x= & \sum_{k=0}^{\infty} \alpha_{k} \sqrt{\pi} \Gamma(k+1)\left(\frac{2}{\omega}\right)^{k+\frac{1}{2}} J_{k+\frac{1}{2}}(\omega) \\
& +i \sum_{k=0}^{\infty} \beta_{k} \sqrt{\pi} \Gamma(k+1)\left(\frac{2}{\omega}\right)^{k+\frac{1}{2}} J_{k+\frac{3}{2}}(\omega) .
\end{aligned}
$$

Remark 1. In Theorem 2, if $\omega<0$, we should rewrite the path as $\gamma=$ $\left\{z \mid z=t-i a\left(1-t^{2}\right),-1 \leq t \leq 1,0 \leq a \leq 1\right\}$. For real number $\omega \neq 0$, it is obvious that

$$
\begin{aligned}
\int_{-1}^{1} f(x) e^{i \omega x} d x= & \sum_{k=0}^{\infty} \alpha_{k} \sqrt{\pi} \Gamma(k+1)\left(\frac{2}{|\omega|}\right)^{k+\frac{1}{2}} J_{k+\frac{1}{2}}(|\omega|) \\
& +i \cdot \operatorname{sign}(\omega) \sum_{k=0}^{\infty} \beta_{k} \sqrt{\pi} \Gamma(k+1)\left(\frac{2}{|\omega|}\right)^{k+\frac{1}{2}} J_{k+\frac{3}{2}}(|\omega|) .
\end{aligned}
$$

Remark 2. We further enlarge the inequality in Equation (8): Firstly, if $n \rightarrow \infty$ with $a=0$ and $\omega$ fixed, the right side of Equation (8) behaves as const $\frac{1}{\sqrt{n}\left(R^{2}-1\right)^{n}} \rightarrow 0$. Secondly, if $\omega \rightarrow+\infty$ with $a=1$ and $n$ fixed,

$$
\left|\rho_{n}\right| \leq \text { const } \int_{-1}^{1}\left(1-t^{2}\right)^{n} e^{-\omega\left(1-t^{2}\right)} d t=O\left(\omega^{-n-1}\right)
$$


In brief, the errors of Equation (7) not only decrease as the order $n$ of the derivatives increases, but also decrease rapidly as the frequency $\omega$ increases.

\section{The Bessel Expansion of Fourier Integral with Symmetric Branch Points}

Let $U=\left\{\left(z_{1}, z_{2}\right) \in \mathbb{C}^{2}:\left|z_{v}\right|<r_{v}, r_{v}>1, v=1,2\right\}$. In this section, we consider the case that there are symmetric branch points $z= \pm 1$ for $f$. Namely, $f(z)=\varphi\left(z_{1}, z_{2}\right), \varphi \in \mathcal{O}(U) \cap C(\bar{U})$ with $z_{1}=z, z_{2}=\left(1-z^{2}\right)^{\frac{1}{p}}, p \in \mathbb{Z}^{+}$.

\subsection{Hermite Series with Symmetric Branch Points}

The function $\varphi$ can be expressed by the multiple Cauchy integral formula

$$
\varphi\left(z_{1}, z_{2}\right)=\frac{1}{(2 \pi i)^{2}} \int_{\Gamma} \frac{\varphi\left(\zeta_{1}, \zeta_{2}\right)}{\left(\zeta_{1}-z_{1}\right)\left(\zeta_{2}-z_{2}\right)} d \zeta_{1} d \zeta_{2}
$$

where $\Gamma$ is the skeleton of $U$, and, if $\left|\frac{1-z_{1}^{2}}{1-\zeta_{1}^{2}}\right|<1,\left|\frac{z_{2}}{\zeta_{2}}\right|<1$ for $\left(z_{1}, z_{2}\right) \in V \subset U,\left(\zeta_{1}, \zeta_{2}\right) \in \Gamma$, then the above integral kernel can be written as

$$
\begin{aligned}
\frac{1}{\left(\zeta_{1}-z_{1}\right)\left(\zeta_{2}-z_{2}\right)}= & -\frac{\zeta_{1}+z_{1}}{\left(1-\zeta_{1}^{2}\right) \zeta_{2}} \sum_{p k_{1}+k_{2} \leq n-1}\left(\frac{1-z_{1}^{2}}{1-\zeta_{1}^{2}}\right)^{k_{1}}\left(\frac{z_{2}}{\zeta_{2}}\right)^{k_{2}} \\
& -\frac{\zeta_{1}+z_{1}}{\left(1-\zeta_{1}^{2}\right) \zeta_{2}} \sum_{p k_{1}+k_{2} \geq n}\left(\frac{1-z_{1}^{2}}{1-\zeta_{1}^{2}}\right)^{k_{1}}\left(\frac{z_{2}}{\zeta_{2}}\right)^{k_{2}} .
\end{aligned}
$$

Since

$$
-\frac{\zeta_{1}+z_{1}}{\left(1-\zeta_{1}^{2}\right) \zeta_{2}} \sum_{p k_{1}+k_{2} \geq n}\left(\frac{1-z_{1}^{2}}{1-\zeta_{1}^{2}}\right)^{k_{1}}\left(\frac{z_{2}}{\zeta_{2}}\right)^{k_{2}}=\frac{z_{2}^{n}\left(1-z_{1}^{2}\right)^{1 / p}}{\zeta_{2}^{n}\left(\zeta_{1}-z_{1}\right)\left[\zeta_{2}\left(1-z_{1}^{2}\right)^{1 / p}-z_{2}\left(1-\zeta_{1}^{2}\right)^{1 / p}\right]^{\prime}},
$$

by variable substitutions $z_{1}=z, z_{2}=\left(1-z^{2}\right)^{\frac{1}{p}}$ and noting that $f(z)=\varphi\left(z_{1}, z_{2}\right)$, we have

$$
\begin{aligned}
f(z)= & -\frac{1}{(2 \pi i)^{2}} \sum_{p k_{1}+k_{2} \leq n-1}\left(1-z^{2}\right)^{k_{1}+k_{2} / p} \int_{\Gamma} \frac{\left(\zeta_{1}+z\right) \varphi\left(\zeta_{1}, \zeta_{2}\right)}{\left(1-\zeta_{1}^{2}\right)^{k_{1}+1} \zeta_{2}^{k_{2}+1}} d \zeta_{1} d \zeta_{2} \\
& +\frac{\left(1-z^{2}\right)^{n / p}}{(2 \pi i)^{2}} \int_{\Gamma} \frac{\varphi\left(\zeta_{1}, \zeta_{2}\right)}{\left(\zeta_{1}-z\right) \zeta_{2}^{n}} \frac{1}{\zeta_{2}-\left(1-\zeta_{1}^{2}\right)^{1 / p}} d \zeta_{1} d \zeta_{2}
\end{aligned}
$$

Letting the coefficients

$$
\begin{aligned}
& \alpha_{k}=\frac{-1}{(2 \pi i)^{2}} \sum_{p k_{1}+k_{2}=k} \int_{\Gamma} \frac{\zeta_{1} \varphi\left(\zeta_{1}, \zeta_{2}\right)}{\left(1-\zeta_{1}^{2}\right)^{k_{1}+1} \zeta_{2}^{k_{2}+1}} d \zeta_{1} d \zeta_{2}, \\
& \beta_{k}=\frac{-1}{(2 \pi i)^{2}} \sum_{p k_{1}+k_{2}=k} \int_{\Gamma} \frac{\varphi\left(\zeta_{1}, \zeta_{2}\right)}{\left(1-\zeta_{1}^{2}\right)^{k_{1}+1} \zeta_{2}^{k_{2}+1}} d \zeta_{1} d \zeta_{2},
\end{aligned}
$$

we can rewrite Equation (11) as Hermite series of $f$,

$$
f(z)=\sum_{k=0}^{n-1} \alpha_{k}\left(1-z^{2}\right)^{\frac{k}{p}}+z \sum_{k=0}^{n-1} \beta_{k}\left(1-z^{2}\right)^{\frac{k}{p}}+\rho_{n}, \quad|z| \leq 1,
$$


where the remainder is

$$
\rho_{n}=\frac{\left(1-z^{2}\right)^{n / p}}{(2 \pi i)^{2}} \int_{\Gamma} \frac{\varphi\left(\zeta_{1}, \zeta_{2}\right)}{\left(\zeta_{1}-z\right) \zeta_{2}^{n}} \frac{1}{\zeta_{2}-\left(1-\zeta_{1}^{2}\right)^{1 / p}} d \zeta_{1} d \zeta_{2}
$$

The coefficients can be computed by the multi-dimension residue theorem. Letting

$$
\sigma_{1}\left(\zeta_{1}, \zeta_{2}\right)=-\frac{\zeta_{1} \varphi\left(\zeta_{1}, \zeta_{2}\right)}{\left(1+\zeta_{1}\right)^{k_{1}+1}}, \quad \sigma_{2}\left(\zeta_{1}, \zeta_{2}\right)=-\frac{\zeta_{1} \varphi\left(\zeta_{1}, \zeta_{2}\right)}{\left(1-\zeta_{1}\right)^{k_{1}+1}}
$$

by multi-Taylor expansion,

$$
\begin{aligned}
& \sigma_{1}\left(\zeta_{1}, \zeta_{2}\right)=\left.\sum_{k_{1}, k_{2}=0}^{\infty} \frac{1}{k_{1} ! k_{2} !} \frac{\partial^{k_{1}+k_{2}} \sigma_{1}}{\partial \zeta_{1}^{k_{1}} \partial \zeta_{2}^{k_{2}}}\right|_{(1,0)}\left(\zeta_{1}-1\right)^{k_{1}} \zeta_{2}^{k_{2}}, \\
& \sigma_{2}\left(\zeta_{1}, \zeta_{2}\right)=\left.\sum_{k_{1}, k_{2}=0}^{\infty} \frac{1}{k_{1} ! k_{2} !} \frac{\partial^{k_{1}+k_{2}} \sigma_{2}}{\partial \zeta_{1}^{k_{1}} \partial \zeta_{2}^{k_{2}}}\right|_{(-1,0)}\left(\zeta_{1}+1\right)^{k_{1}} \zeta_{2}^{k_{2}},
\end{aligned}
$$

then we obtain the coefficients

$$
\alpha_{k}=\sum_{p k_{1}+k_{2}=k} \frac{1}{k_{1} ! k_{2} !}\left(\left.(-1)^{k_{1}+1} \frac{\partial^{k_{1}+k_{2}} \sigma_{1}}{\partial \zeta_{1}^{k_{1}} \partial \zeta_{2}^{k_{2}}}\right|_{(1,0)}+\left.\frac{\partial^{k_{1}+k_{2}} \sigma_{2}}{\partial \zeta_{1}^{k_{1}} \partial \zeta_{2}^{k_{2}}}\right|_{(-1,0)}\right) .
$$

Similarly, letting

$$
\tau_{1}\left(\zeta_{1}, \zeta_{2}\right)=-\frac{\varphi\left(\zeta_{1}, \zeta_{2}\right)}{\left(1+\zeta_{1}\right)^{k_{1}+1}}, \quad \tau_{2}\left(\zeta_{1}, \zeta_{2}\right)=-\frac{\varphi\left(\zeta_{1}, \zeta_{2}\right)}{\left(1-\zeta_{1}\right)^{k_{1}+1}}
$$

we have

$$
\beta_{k}=\sum_{p k_{1}+k_{2}=k} \frac{1}{k_{1} ! k_{2} !}\left(\left.(-1)^{k_{1}+1} \frac{\partial^{k_{1}+k_{2}} \tau_{1}}{\partial \zeta_{1}^{k_{1}} \partial \zeta_{2}^{k_{2}}}\right|_{(1,0)}+\left.\frac{\partial^{k_{1}+k_{2}} \tau_{2}}{\partial \zeta_{1}^{k_{1}} \partial \zeta_{2}^{k_{2}}}\right|_{(-1,0)}\right) .
$$

Theorem 3. For the Hermite series in Equation (12), if $f(z)=\varphi\left(z_{1}, z_{2}\right), \varphi \in \mathcal{O}(U) \cap C(\bar{U})$ with $z_{1}=$ $z, z_{2}=\left(1-z^{2}\right)^{\frac{1}{p}}, p \in \mathbb{Z}^{+}$and $r_{1}>1, r_{2}>\left(1+r_{1}^{2}\right)^{1 / p}$, then we have the error estimation of the remainder

$$
\left|f(z)-\sum_{k=0}^{n-1} \alpha_{k}\left(1-z^{2}\right)^{\frac{k}{p}}-z \sum_{k=0}^{n-1} \beta_{k}\left(1-z^{2}\right)^{\frac{k}{p}}\right| \leq C\left|1-z^{2}\right|^{\frac{n}{p}}, \quad|z| \leq 1,
$$

where

$$
C=\frac{\max _{\left|\zeta_{1}\right|=r_{1},\left|\zeta_{2}\right|=r_{2}}\left|\varphi\left(\zeta_{1}, \zeta_{2}\right)\right|}{\left(r_{1}-1\right) r_{2}^{n-1}} \frac{r_{1}}{r_{2}-\left(1+r_{1}^{2}\right)^{1 / p}} .
$$

Proof. In Equation (12), $\rho_{n}$ can be rewritten as

$$
\rho_{n}=\frac{\left(1-z^{2}\right)^{n / p}}{(2 \pi i)^{2}} \int_{\Gamma} \frac{\varphi\left(\zeta_{1}, \zeta_{2}\right)}{\left(\zeta_{1}-z\right) \zeta_{2}^{n}} \frac{1}{\zeta_{2}-\left(1-\zeta_{1}^{2}\right)^{1 / p}}, d \zeta_{1} d \zeta_{2}
$$

then

$$
\left|\rho_{n}\right| \leq \frac{\max _{\left|\zeta_{1}\right|=r_{1},\left|\zeta_{2}\right|=r_{2}}\left|\varphi\left(\zeta_{1}, \zeta_{2}\right)\right|}{\left(r_{1}-1\right) r_{2}^{n-1}} \frac{r_{1}}{r_{2}-\left(1+r_{1}^{2}\right)^{1 / p}}\left|1-z^{2}\right|^{n / p}, \quad|z| \leq 1
$$


4.2. The Bessel Expansion of Fourier Integral with Symmetric Branch Points

Similar to Theorem 2, we have the Bessel expansion of Fourier integral with symmetric branch points, and the error estimation is as follows.

Theorem 4. For $\omega>0,0 \leq a \leq 1$, under the assumptions of Theorem 3, we have

$$
\begin{aligned}
\int_{-1}^{1} f(x) e^{i \omega x} d x= & \sum_{k=0}^{n-1} \alpha_{k} \sqrt{\pi} \Gamma(k / p+1)\left(\frac{2}{\omega}\right)^{k / p+1 / 2} J_{k / p+1 / 2}(\omega) \\
& +i \sum_{k=0}^{n-1} \beta_{k} \sqrt{\pi} \Gamma(k / p+1)\left(\frac{2}{\omega}\right)^{k / p+1 / 2} J_{k / p+3 / 2}(\omega)+\rho_{n} .
\end{aligned}
$$

where the remainder satisfying

$$
\left|\rho_{n}\right| \leq C_{2}\left(1+4 a^{2}\right)^{(n / p+1) / 2} \int_{-1}^{1}\left(1-t^{2}\right)^{n / p} e^{-\omega a\left(1-t^{2}\right)} d t
$$

and $C_{2}$ is independent of $\omega$ and $a$.

\section{The Numerical Experiments}

In this section, for the two cases of analytic functions and branch functions, we present two examples that verify the validity of Bessel expansion to calculate Fourier integrals. These numerical experiments were done in Maple 16.

Example 1. We consider the case of $f(x)=e^{1-x^{2}}$. According to Equations (7) and (8), we have

$$
\left|\int_{-1}^{1} e^{1-x^{2}} e^{i \omega x} d x-\sqrt{\pi} \sum_{k=0}^{3}\left(\frac{2}{\omega}\right)^{k+1 / 2} J_{k+1 / 2}(\omega)\right| \leq 0.066\left(1+4 a^{2}\right)^{9 / 2} \int_{-1}^{1}\left(1-t^{2}\right)^{4} e^{-\omega a\left(1-t^{2}\right)} d t .
$$

Similarly, we have

$$
\left|\int_{-1}^{1} e^{1-x^{2}} e^{i \omega x} d x-\sqrt{\pi} \sum_{k=0}^{4}\left(\frac{2}{\omega}\right)^{k+1 / 2} J_{k+1 / 2}(\omega)\right| \leq 0.013\left(1+4 a^{2}\right)^{11 / 2} \int_{-1}^{1}\left(1-t^{2}\right)^{5} e^{-\omega a\left(1-t^{2}\right)} d t .
$$

The two sides of the inequalities in Equations (16) and (17) are shown in Figure 2 for $\omega=0.1+2 m, m=$ $0,1, \cdots, 50$. The absolute errors of the expansion on the left side of the inequalities in Equations (16) and (17) are represented by diamond points. Besides, the error estimates on the right of Equations (16) and (17) are represented by dash lines.

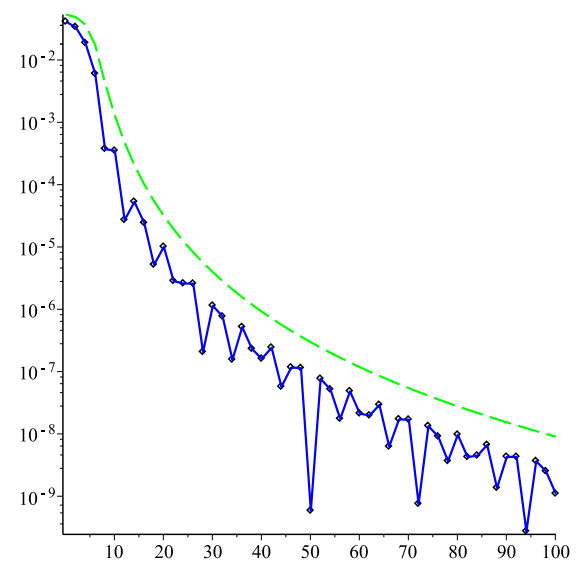

(a) Equation (16)

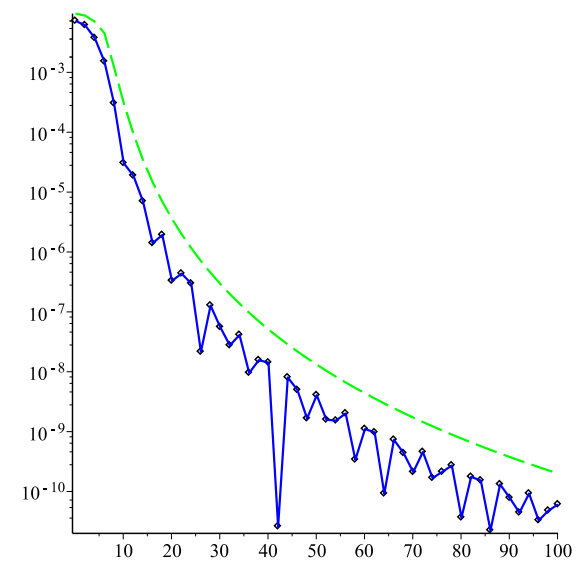

(b) Equation (17)

Figure 2. The errors and their estimation of the expansions for Equations (16) and (17). 
Example 2. We consider the case of $f(x)=e^{\sqrt{1-x^{2}}}$ or $f(x)=e^{x \sqrt{1-x^{2}}}$. According to Equations (14) and (15), we have

$$
\begin{aligned}
& \left|\int_{-1}^{1} e^{\sqrt{1-x^{2}}} e^{i \omega x} d x-\sqrt{\pi} \sum_{k=0}^{4} \frac{\Gamma(k / 2+1)}{k !}\left(\frac{2}{\omega}\right)^{k / 2+1 / 2} J_{k / 2+1 / 2}(\omega)\right| \\
\leq & 0.011\left(1+4 a^{2}\right)^{7 / 2} \int_{-1}^{1}\left(1-t^{2}\right)^{5 / 2} e^{-\omega a\left(1-t^{2}\right)} d t
\end{aligned}
$$

and

$$
\begin{aligned}
& \left|\int_{-1}^{1} e^{x \sqrt{1-x^{2}}} e^{i \omega x} d x-\sqrt{\pi} \sum_{k=0}^{4} \Gamma(k / 2+1)\left(\frac{2}{\omega}\right)^{k / 2+1 / 2}\left(\alpha_{k} J_{k / 2+1 / 2}(\omega)+i \beta_{k} J_{k / 2+3 / 2}(\omega)\right)\right| \\
\leq & 0.25\left(1+4 a^{2}\right)^{7 / 2} \int_{-1}^{1}\left(1-t^{2}\right)^{5 / 2} e^{-\omega a\left(1-t^{2}\right)} d t,
\end{aligned}
$$

where $\alpha_{0}=1, \alpha_{1}=0, \alpha_{2}=1 / 2, \alpha_{3}=0, \alpha_{4}=-11 / 24$ and $\beta_{0}=0, \beta_{1}=1, \beta_{2}=0, \beta_{3}=1 / 6, \beta_{4}=0$. The two sides of the inequalities in Equations (18) and (19) are shown in Figure 3 for $\omega=0.1+2 m, \quad m=$ $0,1, \cdots, 50$. The absolute errors on the left side of Equations (18) and (19) are represented by diamond points. Besides, the error estimates on the right of Equations (18) and (19) are represented by dash lines.

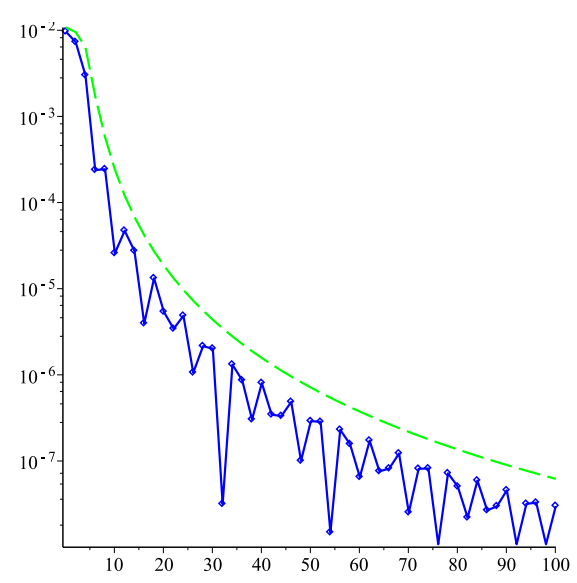

(a) Equation (18)

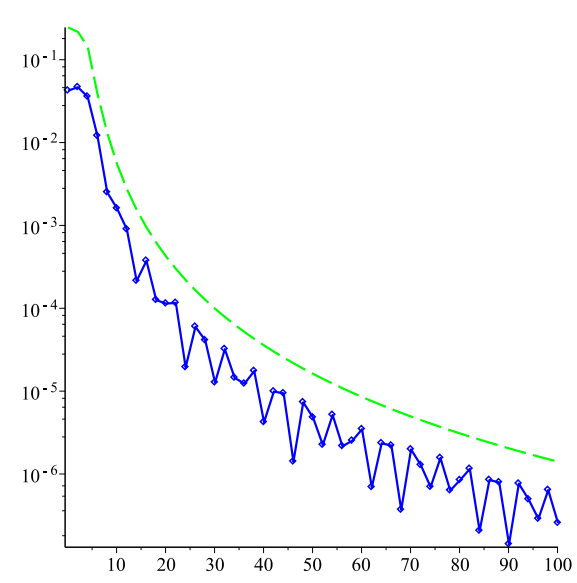

(b) Equation (19)

Figure 3. The errors and their estimation of the expansions for Equations (18) and (19).

\section{The Conclusions and Acknowledgement}

In this article, we make the expression of the Filon-type method more concise by the Bessel expansions for calculating Fourier integral. Moreover, we also derive the convergence rate of the Filon-type method by means of complex analysis. In fact, both error analysis and numerical tests have shown that the Filon-type method is efficient. On the road of frequency analysis and calculation, we will continue to study.

Author Contributions: For methodology, Y.Z. and Z.Z.; software, Y.Z.; validation, Z.Z.; formal analysis, Y.Z. and Z.Z.; writing-original draft preparation, Y.Z.; writing-review and editing, Z.Z.

Funding: This research was funded by project of enhancing school with innovation of Guangdong Ocean University grant number GDOU2016050228 and the APC was funded by project of enhancing school with innovation of Guangdong Ocean University(Q18306).

Acknowledgments: The authors sincerely thank the reviewers for their comments and helpful guidance.

Conflicts of Interest: The authors declare no conflict of interest. 


\section{References}

1. Born, M.; Wolf, E. Principles of Optics; Cambridge University Press: Cambridge, UK, 2001.

2. Watson, G.N. A Treatise on the Theory of Bessel Function; Cambridge University Press: Cambridge, UK, 1966.

3. Deaño, A.; Huybrechs, D.; Iserles, A. Computing Highly Oscillatory Integrals; SIAM: Philadelphia, PA, USA, 2018.

4. Huybrechs, D.; Iserles, A.; Nørsett, S.P. From high oscillation to rapid approximation V: The equilateral triangle. IMA J. Numer. Anal. 2010, 30, 755-785. [CrossRef]

5. Xiang, S.H. Efficient Filon-type methods for $\int_{a}^{b} f(x) e^{i \omega g(x)} d x$. Numer. Math. 2007, 105, 633-658. [CrossRef]

6. Xiang, S.H.; Brunner, H. Efficient methods for Volterra integral equations with highly oscillatory Bessel kernels. BIT Numer. Math. 2013, 53, 241-263. [CrossRef]

7. Kang, H. Efficient calculation and asymptotic expansions of many different oscillatory infinite integrals. Appl. Math. Comput. 2019, 346, 305-318. [CrossRef]

8. Filon, L.N.G. On a quadrature formula for trigonometric integrals. Proc. R. Soc. Edinb. 1928, 49, 38-47. [CrossRef]

9. Gradshteyn, I.S.; Ryzhik, I.M. Table of Integrals, Series, and Products, 7th ed.; Elsevier/Academic Press: Amsterdam, The Netherlands, 2007.

10. Abramowitz, M.; Stegun, I.A. Handbook of Mathematical Functions: With Formulas, Graphs, and Mathematical Tables; Dover Publications Inc.: New York, NY, USA, 1965.

11. Wang, Z.X.; Guo, D.R. Special Functions; World Scientific: Singapore, 1989.

12. Ahlfors, L.V. Complex Analysis: An Introduction to the Theory of Analytic Functions of One Complex Variable; McGraw-Hill Book Company, Inc.: New York, NY, USA; Toronto, ON, Canada; London, UK, 1979.

(C) 2019 by the authors. Licensee MDPI, Basel, Switzerland. This article is an open access article distributed under the terms and conditions of the Creative Commons Attribution (CC BY) license (http:/ / creativecommons.org/licenses/by/4.0/). 